\title{
Bone morphogenetic protein (BMP) receptor inhibitor JL5 synergizes with Ym155 to induce AIF- caspase independent cell death.
}

\section{Arindam Mondal}

Rutgers Robert Wood Johnson Medical School

\section{Rachel NeMoyer}

Rutgers Robert Wood Johnson Medical School

\section{Elaine Langenfeld}

Rutgers Robert Wood Johnson Medical School

\section{Danea Glover}

Rutgers Biomedical and Health Sciences

\section{Michael Scott}

Rutgers Robert Wood Johnson Medical School

\section{Lauren Lairson}

Rutgers The State University of New Jersey

\section{Andrew Zloza}

Rush University Medical Center

\section{David Augeri}

Rutgers University Ernest Mario School of Pharmacy

\section{John Gilleran}

Rutgers University Ernest Mario School of Pharmacy

\section{Youyi Peng}

Rutgers Cancer Institute of New Jersey

\section{Jacques Roberge}

Rutgers University Ernest Mario School of Pharmacy

John E. Langenfeld ( $\square$ langenje@cinj.rutgers.edu )

Rutgers Robert Wood Johnson Medical School

\section{Research article}

Keywords: BMP, Ym155, AIF, cell death, caspase-independent

Posted Date: April 1st, 2020

DOl: https://doi.org/10.21203/rs.3.rs-20532/v1 
License: (c) (i) This work is licensed under a Creative Commons Attribution 4.0 International License. Read Full License 


\section{Abstract}

Background: BMP is an evolutionary conserved morphogen that is reactivated in lung carcinomas. BMP receptor inhibitors promote cell death of lung carcinomas by mechanisms not fully elucidated. The studies here reveal novel mechanisms by which the "survivin" inhibitor Ym155 in combination with the BMP inhibitor JL5 synergistically induces death of lung cancer cells.

Methods: This study examines the mechanism by which Ym155 in combination with JL5 downregulates BMP signaling and induces cell death of non-small cell lung carcinomas (NSCLC) cell lines. Validation experiments were performed on five passage 0 primary NSCLC.

Results: We found that Ym155, which is reported to be a survivin inhibitor, potently inhibits BMP signaling by causing BMPR2 mislocalization into the cytoplasm and its decreased expression. Ym155 mediated cell death is not caused by the inhibition of survivin but involves Ym155 binding to mitochondrial DNA leading to depletion of ATP. The combination of Ym155 and the BMP receptor inhibitor JL5 synergistically causes the downregulation of BMP Smad-1/5 dependent and independent signaling and the induction of cell death of lung cancer cell lines and primary lung tumors. Cell death involves the nuclear translocation of apoptosis inducing factor (AIF) from the mitochondria to the nucleus causing DNA double stranded breaks independent of caspase activation, which occurs only when JL5 and Ym155 are used in combination. Knockdown of BMPR2 together with Ym155 also induced AlF localization to the nucleus.

Conclusions: These studies suggest that inhibition of BMPR2 together with Ym155 can induce AIF caspase-independent cell death. AIF caspase-independent cell is an evolutionary conserved cell death pathway that has never been targeted to induce cell death in cancer cells. These studies provide mechanistic insight how to target AIF caspase-independent cell death using BMP inhibitors.

\section{Background}

The bone morphogenetic proteins are evolutionarily conserved cytokines regulating a plethora of signaling events throughout development. Following the development of the fetal lung there is a decrease in BMP signaling with little activity in the mature lung [1,2]. BMP signaling is reactivated in lung and many other carcinomas [3]. In non-small cell lung carcinomas (NSCLC), the BMP-2 ligand is overexpressed 17-fold higher in comparison to normal lung tissue and benign lung tumors [4]. A number of studies have shown that BMP signaling has significant tumorigenic effects that involve the regulation of cell survival, migration, proliferation, stemness, angiogenesis, and high ligand expression correlates with a worse prognosis $[3,5-10]$.

The BMP ligands signal through receptor serine/threonine kinases [11]. BMP ligands bind to the BMP type 1 receptors (BMPR1) (alk2, alk3, or alk6) promoting phosphorylation by the constitutively active BMP type 2 receptors (BMPR2) (BMPR2, ActR-IIA, ActRIB) [11]. The BMP complex phosphorylates Smad-1/5 that translocates to the nucleus regulating transcriptional events including the inhibitor of differentiation 
protein promoters (Id1, Id2, and Id3) [12-14]. Id1 has tumorigenic effects regulating self-renewal and migration of cancer cells [15-19]. Recent studies suggest that the BMP signaling cascade mediates cell survival independent of its regulation of Smad-1/5 transcription. Inhibition of BMPR2 decreases the expression of the anti-apoptotic factor $X$ chromosome-linked inhibitor of apoptosis protein (XIAP) transforming growth factor beta (TGF $\beta$ ) activated kinase 1 (TAK1) $[7,20,21]$. The inhibition of XIAP is a mechanism by which the BMP inhibitor JL5 creates synergistic cell death in lung cancer cells with TRAIL [22]. Inhibition of BMPR2 and not the type I BMP receptors increases the release of the pro-apoptotic mitochondrial proteins Smac/DIABLO and cytochrome c into the cytosol[22].

Here we report that the BMP receptor inhibitor JL5 and the "survivin" inhibitor Ym155 synergistically promote cell death of lung cancer cell lines and primary tumors. We identified novel mechanism by which Ym155 promotes cell death in cancer cells, which involves the inhibition of BMPR2 Smad-1/5 independent signaling. We also identified that cell death induced by Ym155 involves the binding of Ym155 to mitochondrial DNA. The combination of Ym155 and JL5 synergistically decreases BMP independent signaling by inhibiting BMPR2. Cell death induced by the combination of Ym155 and BMPR2 inhibition occurs independent of caspase activation and involves the translocation of AlF to the nucleus. These studies provide novel insights into how to target BMPR2 smad-independent signaling to induce cell death of lung cancer cells.

\section{Methods}

\section{The Cell culture and reagents}

The A549, H1299, and U1752 lung cancer cells were cultured in Dulbecco's modified Eagle's medium (DMEM, Sigma Aldrich, Saint Louis, MO, USA) with 5\% fetal bovine serum. JL5 and DMH2 were synthesized by David Augeri and John Gilleran, Rutgers School of Pharmacy. Ym155 was purchased from Selleckchem. Z-VAD-FMK was purchased from R\&D Systems. SB-505124 and necrostatin-1 was purchased from Sigma Aldrich (Saint Louis, MO, USA). PicoGreen was obtained from Invitrogen (MA, USA).

\section{Western blot analysis}

Western blot analysis was performed as previously reported [3]. In brief, total cellular protein concentration was determined using BCA method then separated by SDS-PAGE and transferred to nitrocellulose (Schleicher and Schuell, Keene, $\mathrm{NH}$ ). The primary antibodies used were rabbit monoclonal anti-XIAP, rabbit monoclonal anti-Smac/Diablo, rabbit monoclonal anti-cytochrome c, rabbit monoclonal anti-c-IAP1, rabbit monoclonal anti-pTAK1, rabbit monoclonal anti-activated caspase-3, rabbit monoclonal anti-PARP, rabbit monoclonal anti-AIF, rabbit monoclonal anti-pSmad1/5, (Cell signaling Technology, MA, USA), rabbit monoclonal anti-Id1 (Calbioreagents, San Mateo, CA), rabbit polyclonal anti-Smad 1/5 (Upstate Biotechnology, NY, USA), rabbit polyclonal survivin (Novus Biologicals, CO, USA), rabbit antiactin, an affinity isolated antigen specific antibody (Sigma, Saint Louis, MO), and rabbit polyclonal antiGAPDH (Sigma) and mouse monoclonal anti-Spectrin (EMD Millipore, CA, USA). 


\section{Cell viability}

Cells were plated in duplicate into 6-well plates and treated the next day for the designated period of time. Cell counts were determined using the automated cell counter Vi-CELL cell analyzer (Beckman Coulter). Approximately 500 cells per sample were analyzed and trypan blue dye exclusion determined number of dead cells. The experiment was replicated three times in our laboratory.

\section{Transient knockdown}

Validated select siRNA was used to knockdown BMPR2 (Life Technologies). The ID numbers for the siRNA are: BMPR2 (s2044 and s2045). Silencer Select negative control siRNA (4390843) was used to evaluate selectivity. Transfections of the siRNA were performed in duplicate using Lipofectamine ${ }^{\circledR}$ RNAiMAX Reagent (Invitrogen, Carlsbad, CA, USA) according to manufacturer's protocol. Cells were transfected with $6 \mathrm{nM}$ BMPR2 or $6 \mathrm{nM}$ of siRNA control. The experiment was replicated three times in our laboratory.

\section{Cytosol extraction}

Cytosolic protein extraction was performed using Mitochondria/Cytosol fractionation kit as per manufacturer's instructions (Enzo Life Sciences, NY, USA). Cell pellets were resuspended in 100 $\mu$ l of icecold Cytosol Extraction Buffer Mix containing dithiothreitol (DTT) and Protease Inhibitors. After a 10 minute incubation on ice, cells were homogenized. The homogenates were collected to a fresh $1.5 \mathrm{ml}$ tube and centrifuged at $700 \mathrm{xg}$ for $10 \mathrm{~min}$ at $4^{\circ} \mathrm{C}$. The supernatant was collected as the cytosolic fraction and used for further experiments.

\section{TUNEL assay}

DNA double strand breaks (DSB) after treatment were analyzed by using FlowTACS In Situ TUNEL-based apoptosis detection kit (Trevigen) according to the manufacturer's protocol. Cells were treated in duplicate. After treatment, cells were trypsinized and the cell pellet was fixed with $4 \%$ formaldehyde and permeabilized with cytonin for $30 \mathrm{~min}$. Cells were washed with labeling buffer and resuspended in reaction mix for 1 hour $(h)$ then stained with strep-fluorescein solution and analyzed by using flow cytometry (LSRII, BD Biosciences). The experiment was replicated three times in our laboratory.

\section{Immunofluorescence staining}

Cells were seeded for $24 \mathrm{~h}$ onto microscope cover glasses in a 6-well plate then treated. Cells were fixed with $4 \%$ formaldehyde and permeabilized with $0.5 \%$ triton-X. Cells were blocked with CAS-block for $1 \mathrm{~h}$; cells were stained with anti-BMPR2 antibody that recognizes an extracellular epitope (Sigma-Aldrich) or AlF for $1 \mathrm{~h}$ at room temperature. To assess for BMPR2 on the plasma membrane the cells were fixed but not permeabilized prior to staining. Cells were then washed with phosphate buffered saline (PBS) and stained with Alexa Flour 488 conjugated secondary antibody for $1 \mathrm{~h}$ at room temperature. After washing with PBS, the nuclei were counterstained with 4',6-diamidino-2-phenylindole (DAPI) (Sigma-Aldrich) for 10 
min. After drying, cells were observed under a fluorescence microscope (Nikon eclipse TE300). All the immunofluorescence experiments except the primary tumors cells were replicated three times in our laboratory. The AIF immunofluorescence study using primary tumor cell line was performed once for each of the 5 tumors.

\section{PicoGreen staining}

Approximately, 450,000 cells per well were seeded onto microscope coverslips in a 6 well plate for $24 \mathrm{~h}$. After treatment, $3 \mu \mathrm{l} / \mathrm{ml}$ stock PicoGreen solution (Molecular Probes Inc.) was directly added into the culture medium. Cells were then incubated for $1 \mathrm{~h}$ at $37^{\circ} \mathrm{C}$ in $5 \%$ carbon dioxide (CO2). Cells were washed and mounted with ProLong Diamond Antifade Mountant (Invitrogen), dried and examined under a fluorescence microscope (Nikon eclipse TE300). The experiment was replicated two times in our laboratory.

\section{Adenosine triphosphate (ATP) assay}

Trichloroacetic acid (TCA) method for ATP extraction was performed according to the manufacturer's protocol (Enliten $\circledast$ ATP Assay System, Promega). Briefly, 600,000 cells/ well cells were plated and grown overnight at $37^{\circ} \mathrm{C}$ in $5 \%$ carbon dioxide (CO2). Cells were treated in duplicate and after treatment, cell pellets were collected by trypsinization, washed with PBS and transferred into fresh $1.5 \mathrm{ml}$ tubes. The cell suspension was centrifuged at 1500rpm for $5 \mathrm{~min}$. $150 \mu \mathrm{l}$ of ice cold 1\% TCA was added into the cell pellet and mixed for 40 seconds. The mixture was centrifuged at $13000 \mathrm{x} \mathrm{g}$ for $5 \mathrm{~min}$ at $4^{\circ} \mathrm{C}$. The ATP extract was immediately 100 -fold diluted in $1 \mathrm{M}$ tris-acetate buffer $(\mathrm{pH} 7.75)$. Intracellular ATP was measured by Enliten ${ }^{\circledR}$ ATP Assay System using a luminometer (Tecan, Infinite M200Pro). The experiment was replicated three times in our laboratory.

\section{Primary lung carcinomas}

Primary tumor tissues were obtained within 30 min of surgery from The Cancer Institute of New Jersey (CINJ) as approved by the Research Ethics and Institutional Review Board (Protocol Number: 001608). Consecutive non-small cell lung carcinomas were used. The tissues were washed with PBS and then cut into small pieces. Human tissue dissociation kit (Miltenyl Biotec) was used to dissociate the tumor tissues according to the manufacturer's protocol. Dissociated cells were grown in Dulbecco's Modified Eagle Medium (DMEM) media supplemented with 20\% Fetal Bovine Serum (FBS) and 1\% antibioticantimycotic for approximately 10 days without splitting. At $70 \%$ confluence, cells were trypsinized and plated for all the experiments at the same time.

Tumor xenograft studies. Tumor xenografts from H1299 cells were established by injection of 2 million cells into the intradermal space of the flanks of 6 week female NOD-scid IL2Rgammanull (NSG) mice (bred in-house and randomly assigned to groups without blinding). When tumors reached approximately $4 \mathrm{~mm} \times 4 \mathrm{~mm}$, mice were treated with 3 or $10 \mathrm{mg} / \mathrm{kg}$ of JL5 twice daily (or DMSO control) for four days. 
Treated mice were not blinded Experiments were conducted in compliance with ethical regulations and approved by Rutgers IACUC.

\section{Statistical Analysis}

The mean of the control group was compared to the mean of each treated group using a paired student ttest assuming unequal variances. Differences with $p$ values $<0.05$ were considered statistically significant.

\section{Results}

\section{Ym155 decreases BMP signaling}

H1299 cells were injected into the intradermal space of the flanks of NOD SCID IL2Rgammanull (NSG) mice and were treated with DMSO or JL5 for 4 days. We previously reported that JL5 decreases the expression of Id1 but not XIAP in the tumor xenografts [8]. Survivin is in the family of anti-apoptotic proteins that has been reported to be regulated by BMP signaling and is known to stabilize the expression of XIAP [23]. We found for the first time that inhibition of BMP signaling with JL5 causes a significant increase in the expression of survivin in the tumor xenografts (Fig. 1A).

We explored whether Ym155, a reported survivin inhibitor [24], would have synergy when used in combination with a BMP inhibitor. First, we looked at the effects of Ym155 on the expression of survivin and XIAP. Ym155 induced a significant decrease in the expression of XIAP and caused decreased activity in its downstream target TAK1 in H1299 (Fig. 1B) and A549 cells (Fig. 1C) with little change in the expression of survivin. This is consistent with prior studies suggesting that Ym155 mediates its effects independent of survivin [25].

Since BMPR2 regulates XIAP through Smad-1/5 independent mechanisms, we examined whether Ym155 regulated BMP signaling. The effects of Ym155 on BMP Smad-1/5 dependent signaling was by assessed by examining changes in the phosphorylation of the BMP transcription factor Smad-1/5 and its downstream transcriptional target Id1. Ym155 caused a significant decrease in the activity of Smad-1/5 and Id1 in both the H1299 (Fig. 1D) and A549 (Fig. 1E) cells at nanomolar concentrations. H1299 cells were stably transfected with the Id1 promotor driving the expression of the luciferase reporter. Ym155 caused a dose responsive decrease in the Id1 reporter (Fig. 1F). Transfection of constitutively active BMPR1A (caBMPR1A) activates Smad-1/5-Id1 signaling. Ym155 inhibited caBMPR1A activation of Smad-1/5 (Fig. 1G). These studies show that Ym155 regulates both Smad-1/5 dependent and independent signaling, raising the possibility it regulates BMPR2.

\section{Ym155 synergizes with JL5 to decrease BMP signaling and induce cell death}

Since Ym155 does not target the BMP receptors, we examined whether it would induce synergy with JL5. JL5 caused significantly more cell death when used in combination with Ym155 compared to either 
compound alone in three different lung cancer cell lines (Fig. 2A-C). By Western blot analysis, there was no change in BMP signaling with JL5 and Y155 alone or in combination after 3h (Fig. 2D). By 24h, the combination of Ym155 and JL5 significantly decreased the expression of Id1 and XIAP in comparison to each compound alone in both the A549 and H1299 cells (Fig. 2E-F). Ym155 alone induced apoptosis as demonstrated by the expression of activated caspase-3, $17 \mathrm{kd}$ and $19 \mathrm{kd}$ fragments and cleavage of its downstream target poly ADP ribose polymerase (PARP) (Fig. 2G). We found no activation of caspase-3 or caspase-9 when examined at 1, 3, and 24 hours (Fig. $2 \mathrm{G}$ and data not shown). The pan-caspase inhibitor Z-VAD-FMK did not affect cell death induced by JL5 and Ym155 in combination (Fig. 2H). Necrostatin, which inhibits necrosis induced by receptor-associated adaptor kinase 1 (RIP1) [26], also had no effect on cell death induced by JL5 and Ym155 when used in combination (Fig. 2H). These studies suggest that Ym155 together with a BMP receptor inhibitor synergize to mediate cell death by mechanisms independent of caspases or RIP1 induced necrosis.

\section{Ym155 together with JL5 increases cytosolic AIF and Smac/DIABLO}

Since apoptosis is not induced with JL5 and Ym155, we explored whether cell death involved the mitochondrial release of AIF [27]. In cells undergoing AIF induced cell death, AIF is cleaved producing 67 $\mathrm{kd}$ and $57 \mathrm{kd}$ fragments, which is then rapidly transported to the nucleus. Nuclear AIF induces DNA fragmentation and chromatin condensation leading to cell death [27]. The combination of Ym155 and JL5 synergistically enhanced the release of Smac/DIABLO into the cytosol as early as $3 \mathrm{~h}$ and persisted for at least 24h in A549 cells (Fig. 3A-B). In the H1299 cells, we did not identify cytosolic AlF after 3h but was noted $24 \mathrm{~h}$ after being treated with JL5 and Ym155 in combination (Fig. 3C-D). Cytosolic AIF was not observed when JL5 and Ym155 were used alone. Smac/DIABLO causes the inhibition and degradation of anti-apoptotic proteins cellular inhibitor of apoptosis 1 (c-IAP-1). Consistent with an increase in cytosolic Smac/DIABLO, JL5 together with Ym155 caused a significant decrease in the expression of c-IAP-1 (Fig. 3E-F).

\section{BMP inhibitors with YM155 cause DNA double stranded breaks and nuclear localization of AIF}

A hallmark of AIF induced cell death is the induction of DNA double stranded breaks (DSB) and its localization to the nucleus. The TUNEL assay was used to determine DNA-DSB. Three hours following treatment with JL5 or Ym155 alone or in combination no DNA-DSB were found (Figure 4A). After 24h, very few cells treated with JL5 or Ym155 alone demonstrated DNA-DSB. JL5 when used in combination with Ym155 approximately 40\% and 65\% of cells demonstrating DNA-DSB in A549 and H1299 cells, respectively (Fig. 4B). DMH2 is similar to JL5, having potent inhibition of BMP type 1 receptors with some inhibition of BMPR2 [8]. DMH2 treated cells also demonstrated an increase in DNA-DSB when used in combination with Ym155 (Fig. 4C). To assess whether synergy also occurred by inhibiting TGFI signaling, we used the selective TGFQ receptor inhibitor SB-505124 [28]. SB-505124 had no effect on DNA-DSB when used alone or in combination with Ym155 (Figure 4C). Using immunofluorescent imagining, we examined whether AIF localized to the nucleus following treatment with JL5 and Ym155. JL5 and Ym155 alone did not cause the localization of AIF to the nucleus in either the H1299 or A549 
cells (Fig. 4D-G). However, when JL5 was combined with Ym155 there was significant localization of AIF to the nucleus associated with chromatin condensation in both the H1299 and A549 (Fig. 4D-G).

\section{Ym155 decreases BMPR2 expression and promotes BMPR2 mislocalization to the cytoplasm}

Since Ym155 decreases the expression of XIAP and Id1, which are both regulated by BMPR2, we examined if Ym155 regulated expression of BMPR2. Ym155 caused a dose-related decrease in the expression of BMPR2 that was associated with corresponding decrease in the expression of XIAP in both the A549 and H1299 cells (Fig. 5A-B). At lower concentrations of Ym155 (20 nM), which has no effect on the level of BMPR2 expression, there was an enhanced decrease in expression of BMPR2 and XIAP when Ym155 is used in combination with JL5 (Fig. 5C-D). As previously reported, JL5 caused the mislocalization of BMPR2 to the cytoplasm (Fig. 5E) [22]. We show for the first time that Ym155 alone also causes an increase in the localization of BMPR2 to the cytosol (Fig. 5E), which was greatly increased when JL5 and Ym155 were used in combination (Fig. 5E).

To assess whether the increased nuclear localization of AIF in cells treated with JL5 in combination with Ym155 was mediated by then inhibition of BMPR2, we knocked down the expression of BMPR2 with siRNA in H1299 cells then treated the cells with a low concentration of Ym155 (20 nM). The knockdown of BMPR2 together with Ym155 (20 nM) significantly enhanced AlF localization to the nucleus in comparison to siRNA control cells treated with Ym155 (20 nM) (Fig. 5F-G). These studies show that Ym155 decreases BMP signaling, which involves the downregulation of the expression of BMPR2 expression and its mislocalization to the cytoplasm. The data also suggests that the inhibition of BMPR2 is required for AIF localizing to the nucleus.

\section{Ym155 is a mitochondrial DNA binding agent}

A previous report demonstrated that Ym155 promoted a DNA damage response at a significantly lower dose, decreasing the expression of survivin. This study concluded that Ym155 is a chromosomal DNA damaging agent and the effects on survivin are a secondary event [25]. DNA binding agents are widely used as chemotherapeutic agents. DNA binding drugs predominately accumulate in chromosomal DNA, however, some exert their anti-cancer effects by intercalating mitochondrial DNA [29]. Chemotherapeutic agents binding with mDNA have been shown to interfere with mitochondrial function leading to a depletion of ATP. Quenching the fluorescent signaling of DNA binding agents with propidium bromide and Hoescht 33342 have been used to monitor accumulation of DNA binding drugs. PicoGreen, which selectively labels both nuclear and mDNA is used to monitor the accumulation of drugs in mDNA [29].

We utilized PicoGreen quenching studies to assess if Ym155 accumulates in mDNA in the A549 cells. PicoGreen fluorescent signaling was found in the nucleus and cytoplasm of cells, suggesting it bound mDNA (Fig. 6A). Dual immunfluorescent imaging of PicoGreen with the mitochondrial marker TUMF1 showed co-localization of PicoGreen with TUMF1, confirming PicoGreen accumulation in mDNA (Fig. 6A). Increasing concentrations of $\mathrm{Ym} 155$ showed a dose-responsive quenching of PicoGreen fluorescence in the mDNA (Fig. 6B-C). 
To assess whether Ym155 may affect mitochondrial function directly, we examined whether ATP was depleted in $\mathrm{H} 1299$ cells following treatment with Ym155. Low dose of Ym155 (20 nM) caused a small decrease in ATP levels at 3h, which after 24h was substantially lower than DMSO (Fig. 6D). Ym155 caused a dose-related decrease in ATP levels as early as 3h in H1299 cells (Fig. 6E). These studies show for the first time that anti-cancer effects of Ym155 involve binding mDNA causing mitochondrial dysfunction.

\section{Synergy of Ym155 and JL5 in primary NSCLC}

Primary NSCLC were obtained directly from 5 surgically resected lung tumors. Tumors 1, 2, 3 and 5 were adenocarcinomas and tumor 4 was a squamous carcinoma. Tumors were immediately gently digested and plated for cell culture. After approximately 10 days the cells they were treated with JL5 and Ym155 alone and in combination for $48 \mathrm{~h}$. Primary cancer cells were examined for changes in cell survival, regulation of BMP signaling, and nuclear localization of AIF.

The combination of JL5 and Ym155 caused an increase in cell death compared to DMSO control in 5 of 5 tumors (Fig. S5A). In 3 of 5 tumors, the combination of JL5 and Ym155 induced cell death that was greater than either compound alone (Fig. S5A). XIAP was utilized as a marker of the downregulation of BMPR2 Smad-1/5 independent signaling. The combination of JL5 and Ym155 synergistically decreased the expression of XIAP in 3 of 5 tumors (tumors 1-3), which corresponded to the tumors that JL5 in combination with Ym155 resulted in enhanced cell death (Fig. S5A-B). In tumors 3 and 4, Ym155 alone caused a decrease in the expression of XIAP that was not enhanced further with JL5 (Fig. S5A-B).

AIF nuclear localization was examined in primary lung tumors 2-5. AlF nuclear staining was not seen in primary tumors treated with DMSO or JL5 (Fig. S5C-D). Only a small percentage of cells from the primary lung tumors treated with Ym155 demonstrated nuclear staining with AIF (0-10\%). AIF nuclear staining occurred predominantly in primary tumors that demonstrated synergistic cell death with the combination of JL5 and Ym155 (tumors 2 and 3,67\% and 25\% of cells, respectively) (Fig. S5C-D). Nuclei that stained with AIF showed chromosome condensation and were smaller (Fig. S5C, tumor 2). Tumors that did not show synergy with JL5/Ym155 (tumors 4 and 5), the nuclei were smaller but did not stain with AIF (Fig. S5C, tumor 4).

\section{Discussion}

Our prior studies suggested that targeted inhibition of BMPR2 induces cell death mechanisms not seen with the inhibition of BMP type 1 receptors [22]. Knockdown of BMPR2 decreases the expression of XIAP in lung cancer cell lines and increases cytosolic cytochrome $c$ and Smac/DIABLO [22]. The BMP inhibitors JL5 and DMH2 decrease the expression of XIAP and cause more cell death than the BMP inhibitors DMH1 and LDN $[7,8]$. JL5 and DMH2 demonstrate some inhibition of BMPR2 in in vitro phosphorylation kinase assays with an IC50 of approximately $8 \mu \mathrm{M}$ [8]. Despite JL5 having a relatively low IC50 for BMPR2 it causes its mislocalization to the cytoplasm at $2.5 \mu \mathrm{M}$, which does not occur with BMP inhibitors DMH1 or LDN, which have no activity for BMPR2 [22]. The over-expression of XIAP attenuates 
cell death induced by JL5 [22]. These studies suggest that cell death induced by JL5 and/or DMH2 involves the inhibition of BMPR2 and the downregulation of XIAP [22].

In tumor xenograft studies, JL5 caused some tumor regression but when tumors were examined after 21 days there was little cell death [8]. Tumors examined after 4 days of treatment showed that Id1 expression was decreased but there was no decrease in the expression of XIAP [8]. This suggested that signaling pathways were induced that stabilized the expression of XIAP and/or BMPR2 was not sufficiently inhibited. We show in the present study that the expression of survivin is significantly increased in tumor xenografts treated with JL5. Survivin expression is increased by BMP signaling [30] and is known to increase XIAP stability against ubiquitin-dependent degradation [31]. Surprisingly, Ym155, which is reported to be a survivin inhibitor [24], caused a significant decrease in Smad-dependent and independent signaling at nanomolar concentrations. We show that Ym155 causes a significant decrease in the expression of BMPR2 and its mislocalization to the cytoplasm, which are likely mechanisms leading to the decrease in BMP signaling. In lung cancer cells, the BMP type 1 receptors only regulate Smad-dependent signaling while BMPR2 regulates both Smad-dependent and independent BMP signaling. The mechanism by which Ym155 causes a decrease in BMPR2 expression is not known but may involve the trafficking of BMPR2 to the lysosome and subsequent degradation.

Our studies suggest that inhibition of BMPR2 together with Ym155 promotes AlF caspase-independent cell death. AIF is an evolutionary conserved protein that has two independent functions, biogenesis of the electron transport chain and cell death [32-34]. An increase in permeability of the outer mitochondrial membrane (OMM) is also required for its release into the cytosol [35]. AlF is transported to the nucleus where it induces large-scale DNA fragmentation and cell death [27, 34, 35]. AIF caspase-independent cell death occurs in response to ischemic-reperfusion injury in neurons and myocardium and has only infrequently been reported in cancer cells.

Ym155 was originally reported to be a survivin inhibitor [24]. A subsequent study showed that $Y m 155$ resulted in dose-dependent induction of $\mathrm{yH} 2 \mathrm{AX}$ and pKAP1, which are markers of DNA damage, at a concentration lower than required to decrease the expression of survivin [25]. This report concluded that Ym155 is a DNA damaging agent and the suppression of survivin is a secondary event. The mechanisms by which Ym155 induces DNA damage were not examined in this paper. DNA binding agents used in cancer therapeutics cause cell death by intercalating chromosomal DNA, leading to interruption of cell division. Ethidium Bromide and Doxorubicin induction of cell death has also been shown to occur by binding mDNA [29]. We show that Ym155 binds to mDNA disrupting mitochondrial function and contributes to its anti-cancer effects.

\section{Conclusions}

We demonstrate that Ym155 binds mDNA leading to ATP depletion and causes significant downregulation of BMPR2 signaling in lung cancer cells. We show that BMPR2 signaling can be 
synergistically targeted by using a BMPR2 inhibitor and Ym155. The cell death induced by Ym155 and BMPR2 inhibition involves AIF nuclear localization. These studies provide further insight on how target BMP signaling as a therapeutic in cancer and further supports drug development of a more specific and potent BMPR2 inhibitors.

\section{Abbreviations}

Bone morphogenetic protein (BMP); Bone morphogenetic protein receptor 2 (BMPR2); apoptosis inducing factor (AIF); X-linked inhibitor of apoptosis protein (XIAP); Transforming growth factor beta (TGF $\beta$ ) activated kinase 1 (TAK1); Reactive oxygen species (ROS); Including tumour-necrosis factor (TNF)-related apoptosis-inducing ligand (TRAIL); Non-small cell lung carcinomas (NSCLC); Z-VAD-FMK (VAD); LDN193189 (LDN); DNA double stranded breaks (DNA-DSB); inhibitor of apoptosis proteins (c-IAP); mitochondrial DNA (mDNA); NOD SCID IL2Rgammanull (NSG); poly ADP ribose polymerase (PARP); receptor-associated adaptor kinase 1 (RIP1);

\section{Declarations}

\section{Ethics of approval and consent to participate}

Institutional review board of Rutgers University of New Jersey approved tissue to be obtained from patients resected tumors. Consent was obtained from a honest broker who kept patient personal information anonymous.

\section{Consent for publication}

Our manuscript does not contain any individual person's data in any form.

\section{Availability of data and materials}

The datasets obtained and analyzed for this study will be made available from the corresponding author in a reasonable request.

\section{Competing interests}

A provisionary patent application was sent for BMP inhibitor JL5. The full patent application for JL5 has been withdrawn and is no longer being pursued. There are no active or pending financial agreements regarding JL5 nor has any money been received or is pending.

\section{Funding}

This work was supported by grants National Institute of Health R01 CA225830-01A1. The NIH had no role in the design, collection, or analysis of the data in this manuscript. 
Conception and design: $\mathrm{JL}$

Design and synthesis of JL5 and DMH2: DA, JG, JR

Development of methodology: AM, EL

Acquisition of data: $A M, E L, R N, D G, M S, L L, J G, D A, A Z$

Analysis of data: JL, AM, RN, YP

Writing, review, and/or revision of the manuscript: JL, RN, AM.

All authors have read and approved the manuscript

\section{Acknowledgments}

We want to acknowledge the Rutgers Cancer Institute of New Jersey Biomedical Informatics Shared Resource with funding from NCI-CCSG P30CA072720, and the computational resources made possible through the access to the Perceval Linux cluster operated by OARC under NIH 1S100D012346-01A1

\section{Disclosure and Potential Conflicts of Interest}

The authors have no active conflicts of interests with the material in this manuscript. A patent has been issued for $\mathrm{DMH} 2$ but this compound is no longer being pursued for further development by any of the authors.

\section{References}

1. Sountoulidis A, Stavropoulos A, Giaglis S, Apostolou E, Monteiro R, Chuva de Sousa Lopes SM, Chen H, Stripp BR, Mummery C, Andreakos E, Sideras P: Activation of the Canonical Bone Morphogenetic Protein (BMP) Pathway during Lung Morphogenesis and Adult Lung Tissue Repair. PLoS One 2012, 7:e41460. Epub 42012 Aug 41420.

2. Weaver M, Yingling JM, Dunn NR, Bellusci S, Hogan BL: Bmp signaling regulates proximal-distal differentiation of endoderm in mouse lung development. Development 1999, 126:4005-4015.

3. Langenfeld EM, Calvano SE, Abou-Nukta F, Lowry SF, Amenta P, Langenfeld J: The mature bone morphogenetic protein- 2 is aberrantly expressed in non-small cell lung carcinomas and stimulates tumor growth of A549 cells. Carcinogenesis 2003, 24:1445-1454. Epub 2003 Jun 1419.

4. Langenfeld EM, Bojnowski J, Perone $\mathrm{J}$, Langenfeld $\mathrm{J}$ : Expression of bone morphogenetic proteins in human lung carcinomas. Ann Thorac Surg 2005, 80:1028-1032.

5. Langenfeld EM, Langenfeld J: Bone morphogenetic protein-2 stimulates angiogenesis in developing tumors. Mol Cancer Res 2004, 2:141-149.

6. Langenfeld E, Deen M, Zachariah E, Langenfeld J: Small molecule antagonist of the bone morphogenetic protein type I receptors suppresses growth and expression of Id1 and Id3 in lung 
cancer cells expressing Oct4 or nestin. Mol Cancer 2013, 12:129.

7. Augeri DJ, Langenfeld E, Castle M, Gilleran JA, Langenfeld J: Inhibition of BMP and of TGFbeta receptors downregulates expression of XIAP and TAK1 leading to lung cancer cell death. Mol Cancer 2016, 15:27.

8. Newman JH, Augeri DJ, NeMoyer R, Malhotra J, Langenfeld E, Chesson CB, Dobias NS, Lee MJ, Tarabichi S, Jhawar SR, et al: Novel bone morphogenetic protein receptor inhibitor JL5 suppresses tumor cell survival signaling and induces regression of human lung cancer. Oncogene 2018.

9. Owens P, Pickup MW, Novitskiy SV, Giltnane JM, Gorska AE, Hopkins CR, Hong CC, Moses HL: Inhibition of BMP signaling suppresses metastasis in mammary cancer. Oncogene 2015, 34:24372449.

10. Le Page C, Puiffe ML, Meunier L, Zietarska M, de Ladurantaye M, Tonin PN, Provencher D, MesMasson AM: BMP-2 signaling in ovarian cancer and its association with poor prognosis. J Ovarian Res 2009, 2:4.

11. Nickel J, Sebald W, Groppe JC, Mueller TD: Intricacies of BMP receptor assembly. Cytokine Growth Factor Rev 2009, 20:367-377.

12. Hollnagel A, Oehlmann V, Heymer J, Ruther U, Nordheim A: Id genes are direct targets of bone morphogenetic protein induction in embryonic stem cells. J Biol Chem 1999, 274:19838-19845.

13. Katagiri T, Imada M, Yanai T, Suda T, Takahashi N, Kamijo R: Identification of a BMP-responsive element in Id1, the gene for inhibition of myogenesis. Genes Cells 2002, 7:949-960.

14. Korchynskyi $\mathrm{O}$, ten Dijke $\mathrm{P}$ : Identification and functional characterization of distinct critically important bone morphogenetic protein-specific response elements in the Id1 promoter. $J$ Biol Chem 2002, 277:4883-4891.

15. Lyden D, Young AZ, Zagzag D, Yan W, Gerald W, O'Reilly R, Bader BL, Hynes RO, Zhuang Y, Manova K, Benezra R: Id1 and Id3 are required for neurogenesis, angiogenesis and vascularization of tumour xenografts. Nature 1999, 401:670-677.

16. Cheng YJ, Tsai JW, Hsieh KC, Yang YC, Chen YJ, Huang MS, Yuan SS: Id1 promotes lung cancer cell proliferation and tumor growth through Akt-related pathway. Cancer, 307:191-199. Epub 2011 May 2014.

17. Gupta GP, Perk J, Acharyya S, de Candia P, Mittal V, Todorova-Manova K, Gerald WL, Brogi E, Benezra $\mathrm{R}$, Massague J: ID genes mediate tumor reinitiation during breast cancer lung metastasis. Proc Nat/ Acad Sci U S A 2007, 104:19506-19511.

18. Qi X, Li TG, Hao J, Hu J, Wang J, Simmons H, Miura S, Mishina Y, Zhao GQ: BMP4 supports selfrenewal of embryonic stem cells by inhibiting mitogen-activated protein kinase pathways. Proc Natl Acad Sci U S A 2004, 101:6027-6032. Epub 2004 Apr 6029.

19. O'Brien CA, Kreso A, Ryan P, Hermans KG, Gibson L, Wang Y, Tsatsanis A, Gallinger S, Dick JE: ID1 and ID3 regulate the self-renewal capacity of human colon cancer-initiating cells through p21. Cancer Cel/ 2012, 21:777-792. doi: 710.1016/j.ccr.2012.1004.1036. 
20. Liu Z, Shen J, Pu K, Katus HA, Ploger F, Tiefenbacher CP, Chen X, Braun T: GDF5 and BMP2 inhibit apoptosis via activation of BMPR2 and subsequent stabilization of XIAP. Biochim Biophys Acta 2009, 1793:1819-1827.

21. Yamaguchi K, Nagai S, Ninomiya-Tsuji J, Nishita M, Tamai K, Irie K, Ueno N, Nishida E, Shibuya H, Matsumoto K: XIAP, a cellular member of the inhibitor of apoptosis protein family, links the receptors to TAB1-TAK1 in the BMP signaling pathway. Embo j 1999, 18:179-187.

22. NeMoyer R, Mondal A, Vora M, Langenfeld E, Glover D, Scott M, Lairson L, Rongo C, Augeri DJ, Peng $Y$, et al: Targeting bone morphogenetic protein receptor 2 sensitizes lung cancer cells to TRAIL by increasing cytosolic Smac/DIABLO and the downregulation of X-linked inhibitor of apoptosis protein. Cell Commun Signal 2019, 17:150.

23. Dohi T, Okada K, Xia F, Wilford CE, Samuel T, Welsh K, Marusawa H, Zou H, Armstrong R, Matsuzawa S, et al: An IAP-IAP complex inhibits apoptosis. J Biol Chem 2004, 279:34087-34090.

24. Nakahara T, Kita A, Yamanaka K, Mori M, Amino N, Takeuchi M, Tominaga F, Hatakeyama S, Kinoyama I, Matsuhisa A, et al: YM155, a novel small-molecule survivin suppressant, induces regression of established human hormone-refractory prostate tumor xenografts. Cancer Res 2007, 67:8014-8021.

25. Glaros TG, Stockwin LH, Mullendore ME, Smith B, Morrison BL, Newton DL: The "survivin suppressants" NSC 80467 and YM155 induce a DNA damage response. Cancer Chemother Pharmacol 2012, 70:207-212.

26. Degterev A, Hitomi J, Germscheid M, Ch'en IL, Korkina O, Teng X, Abbott D, Cuny GD, Yuan C, Wagner G, et al: Identification of RIP1 kinase as a specific cellular target of necrostatins. Nat Chem Biol 2008, 4:313-321.

27. Susin SA, Lorenzo HK, Zamzami N, Marzo I, Snow BE, Brothers GM, Mangion J, Jacotot E, Costantini $\mathrm{P}$, Loeffler $\mathrm{M}$, et al: Molecular characterization of mitochondrial apoptosis-inducing factor. Nature 1999, 397:441-446.

28. Vogt J, Traynor R, Sapkota GP: The specificities of small molecule inhibitors of the TGFss and BMP pathways. Cell Signal 2011, 23:1831-1842. doi: 1810.1016/j.cellsig.2011.1806.1019. Epub 2011 Jun 1829.

29. Ashley N, Poulton J: Mitochondrial DNA is a direct target of anti-cancer anthracycline drugs. Biochem Biophys Res Commun 2009, 378:450-455.

30. Yang S, Lim M, Pham LK, Kendall SE, Reddi AH, Altieri DC, Roy-Burman P: Bone morphogenetic protein 7 protects prostate cancer cells from stress-induced apoptosis via both Smad and C-Jun NH2terminal kinase pathways. Cancer Res 2006, 66:4285-4290.

31. Altieri DC: Survivin and IAP proteins in cell-death mechanisms. Biochem J 2010, 430:199-205.

32. Wang X, Yang C, Chai J, Shi Y, Xue D: Mechanisms of AlF-mediated apoptotic DNA degradation in Caenorhabditis elegans. Science 2002, 298:1587-1592.

33. Norberg E, Orrenius S, Zhivotovsky B: Mitochondrial regulation of cell death: processing of apoptosisinducing factor (AIF). Biochem Biophys Res Commun 2010, 396:95-100. 
34. Lorenzo HK, Susin SA, Penninger J, Kroemer G: Apoptosis inducing factor (AIF): a phylogenetically old, caspase-independent effector of cell death. Cell Death Differ 1999, 6:516-524.

35. Bano D, Prehn JHM: Apoptosis-Inducing Factor (AIF) in Physiology and Disease: The Tale of a Repented Natural Born Killer. EBioMedicine 2018, 30:29-37.

\section{Supplementary Figure Legends}

Figure S1. Original immunoblots from Figure 1.

Figure S2. Original immunoblots from Figure 2.

Figure S3. Original immunoblots from Figure 3.

Figure S4. Original immunoblots from Figure 5.

Figure S5. JL5 and Ym155 enhance cell death of primary lung tumors. Five NSCLC samples resected from patients were gently digested and placed into cell culture. When the cells reached confluency (approximately 10 days) they were split and the next day treated with JL5 $2.5 \mu \mathrm{M}$ and Ym155 $20 \mathrm{nM}$ alone and in combination for 48 hours. (A) The percent dead cells after treatment. Data depicts the average of two experiments. (B) Western blot analysis of tumors 1-5. (C) Immunofluorescent imaging for AIF (green) and counterstained with DAPI (blue) for tumors 2 and 4. (D) The percentage of number of cells with nuclear staining with AIF after treatment for 48 hours. Approximately 50 epithelial cells were counted per tumor for each condition. Scale bar is equal to $10 \mu \mathrm{m}$. Original Western blots are shown in Supplementary Figure 6.

Figure S6. Original immunoblots from Figure S5.

\section{Figures}




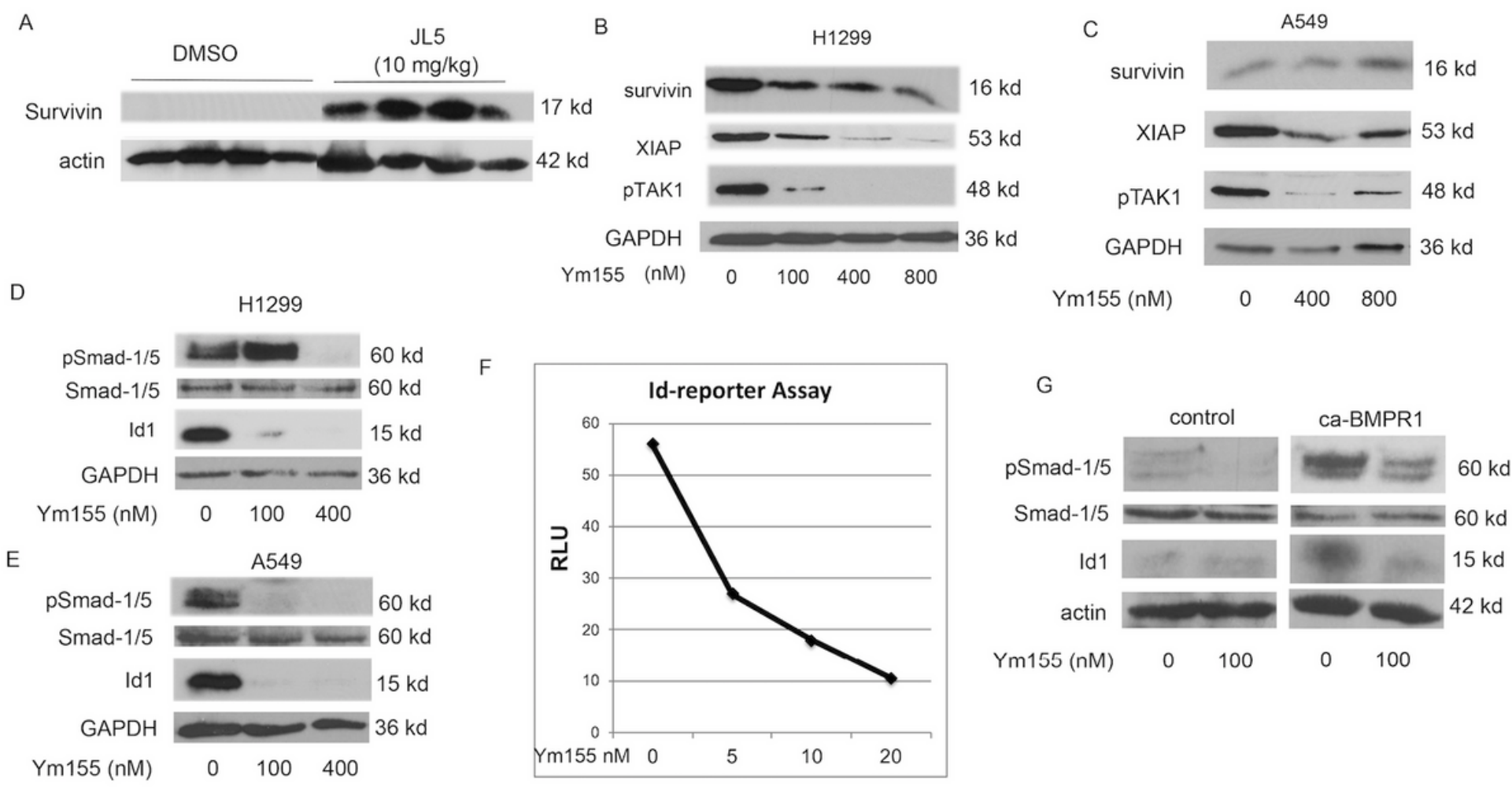

\section{Figure 1}

Ym155 regulates BMP signaling. H1299 cells were injected intradermally into NSG mice. When tumors reached approximately $0.5 \mathrm{~mm} 2$, mice were treated with DMSO or JL5 for 4 days. (A) Western blot analysis shows JL5 treated tumors had a significant increase in the expression of survivin. (B-C) H1299 and A549 cells were treated with Ym155 for 24 hours. Western blot shows Ym155 causes a decrease in Smad 1/5 independent signaling as demonstrated by a decrease in expression of XIAP and TAK activity. (D-E) Western blot showing Ym155 causes a decrease in Smad 1/5 dependent signaling in both the (D) H1299 and (E) A549 cells. (F) H1299 cells stably transfected with Id1-luciferase reporter were treated with Ym155 for 24 hours and relative luminescence units (RVU) measured. Data shown is the mean of 2 independent experiments. (G) H1299 cells were transiently transfected with control vector or caBMPR1 then treated with Ym155 for 24 hours. Western blot analysis showing Ym155 inhibits caBMPR1 activation of Smad-1/5 signaling. Original Western blots are shown in Supplementary Figure 1. Lanes were removed from original Western blots in figure 1A and Figure 1G using Adobe Photo shop as shown in Supplementary Figure 1. 
A

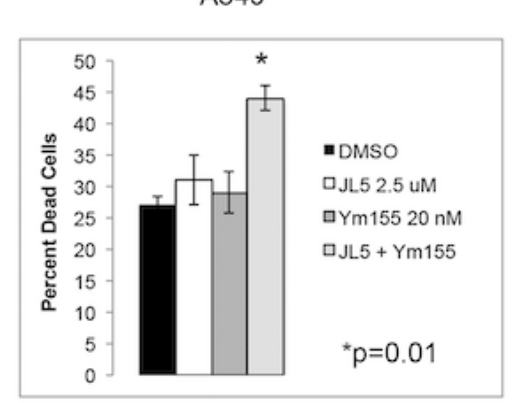

D

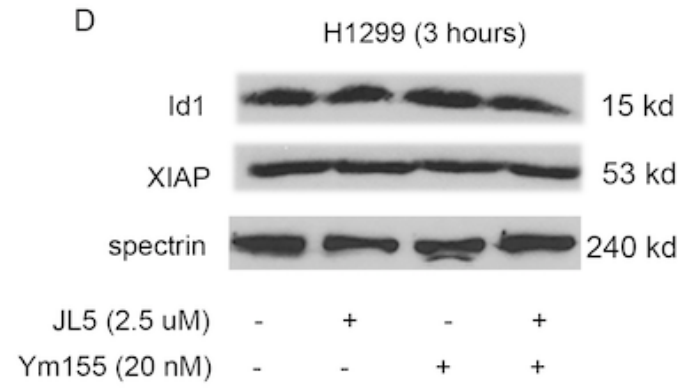

B

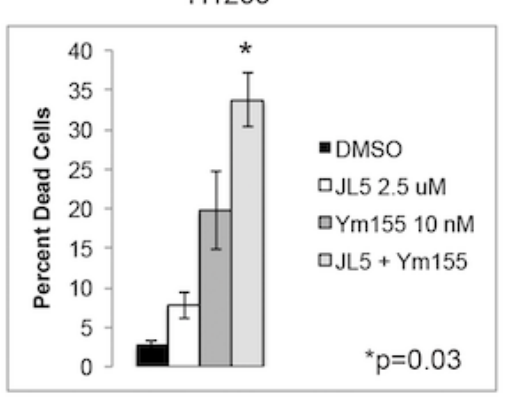

E

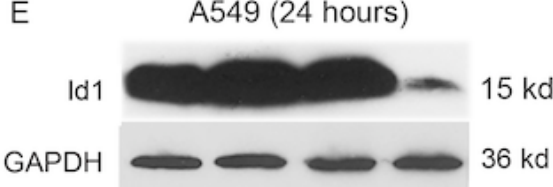

: XIAP

spectrin

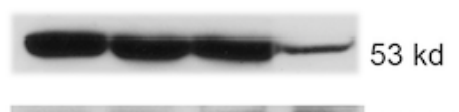

C

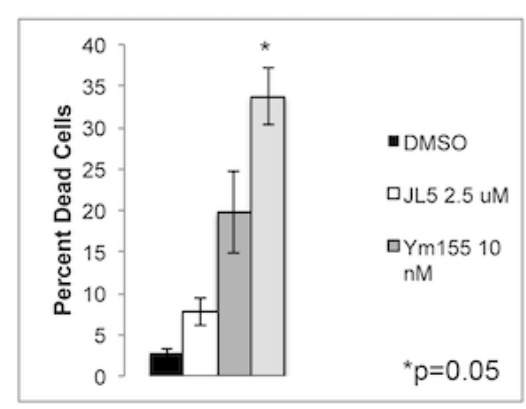

F

ld1

H1299 (24 hours)

GAPDH $15 \mathrm{kd}$ $36 \mathrm{kd}$

JL5 (2.5 uM)
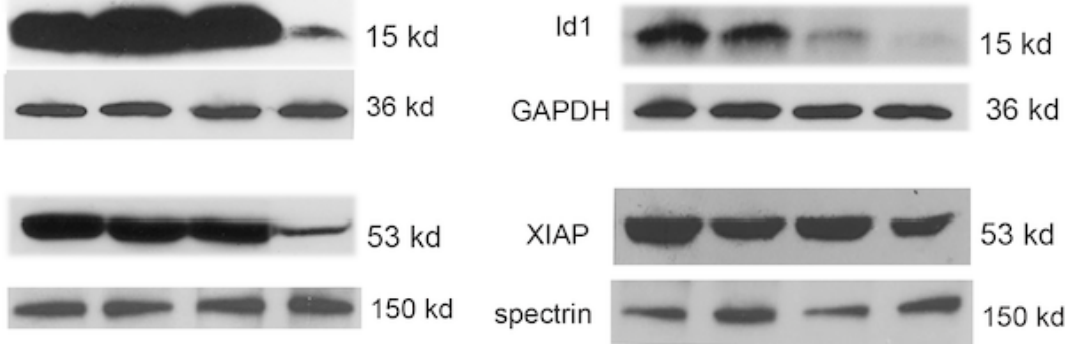

Ym155 (20 nM)

G

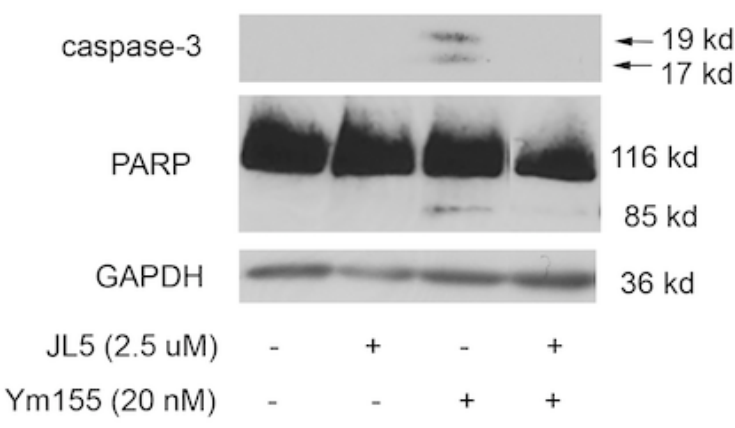

H

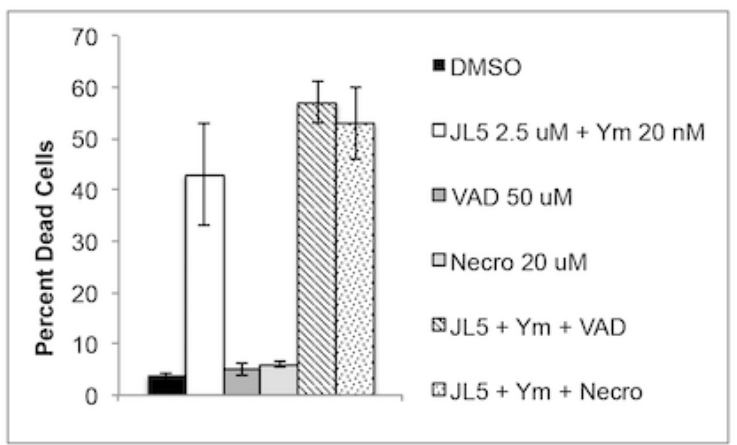

\section{Figure 2}

Ym155 synergizes with JL5. (A-C) Lung cancer cells were treated with Ym155 and JL5 alone and in combination for 24 hours and cell counts performed. (A-B) Represent the mean of four independent experiments and (C) the mean of two experiments. (D-F) Western blot analysis assessing the BMP signaling cascade in response to cells treated with Ym155 and JL5 alone and in combination for 3 or 24 hours. (G) Western blot analysis of H1299 cells treated for 24 hours. Activated caspase-3 and PARP cleavage occurred in cells treated with Ym155. $(\mathrm{H})$ The mean percentage of dead H1299 cells after treatment with JL5 and Ym155 in combination for 24 hours with and without Z-VAD-FMK or necrostatin. Data represents the mean of 4 independent experiments. Original Western blots are shown in Supplementary Figure 2. Lanes were removed from original Western blots in figure $2 \mathrm{G}$ using Adobe Photo shop as shown in Supplementary Figure 2. 
A

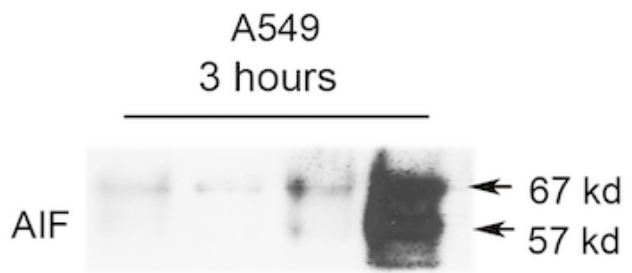

Smac/Diablo

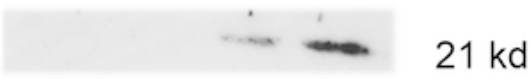

GAPDH

JL5 (2.5 uM)

Ym155 (20 nM)

C

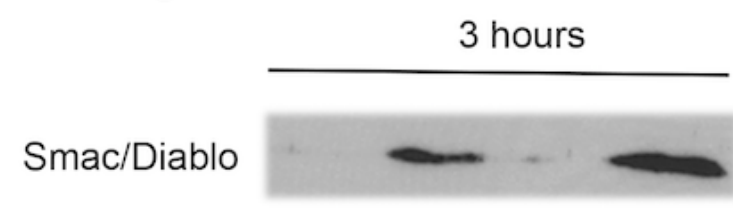

GAPDH

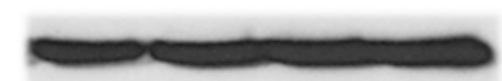

JL5 (2.5 uM)

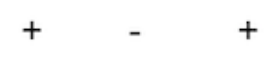

Ym155(20 nM) - - $\quad+\quad+$

E

H1299

C-IAP-1

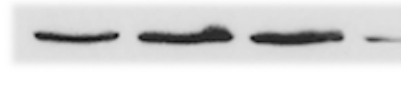

GAPDH

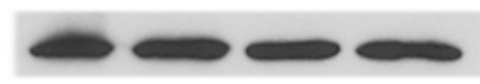

DMSO

JL5 (2.5 UM)

Ym155 (20 nM)
$21 \mathrm{kd}$

$36 \mathrm{kd}$

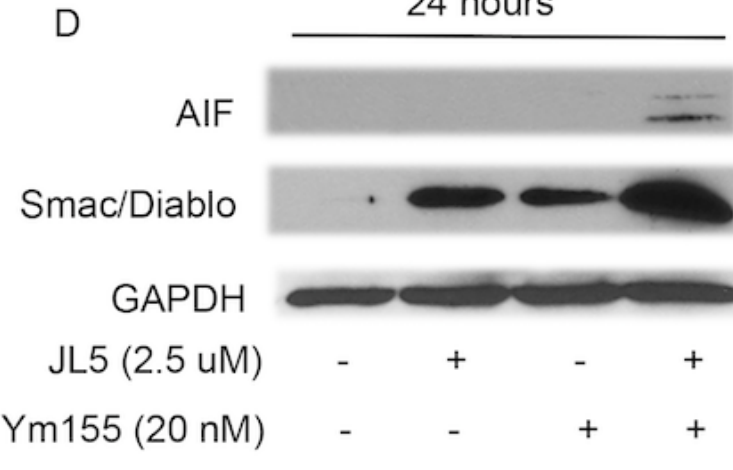

F

$62 \mathrm{kd}$

$36 \mathrm{kd}$

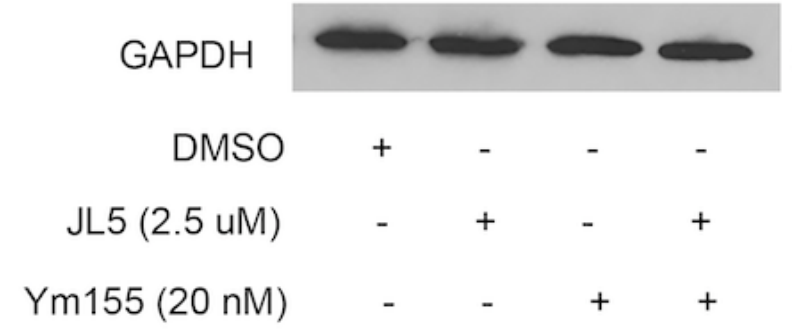

$62 \mathrm{kd}$

$36 \mathrm{kd}$

\section{Figure 3}

Ym155 in combination with JL5 increases cytosolic AIF. (A-D) Western blot of cytosol of A549 and H1299 cells treated with JL5 and Ym155 alone and in combination for 3 or 24 hours. Arrowheads demonstrate the $67 \mathrm{kd}$ and $57 \mathrm{kd}$ fragments of AIF, which only occur in cells treated with JL5 and Ym155 in combination. (E-F) Western blot analysis of cells treated for 24 hours demonstrating a decrease 
expression of anti-apoptotic proteins when cells are treated with both JL5 and Ym155 in combination. Original Western blots are shown in Supplementary Figure 3.

A

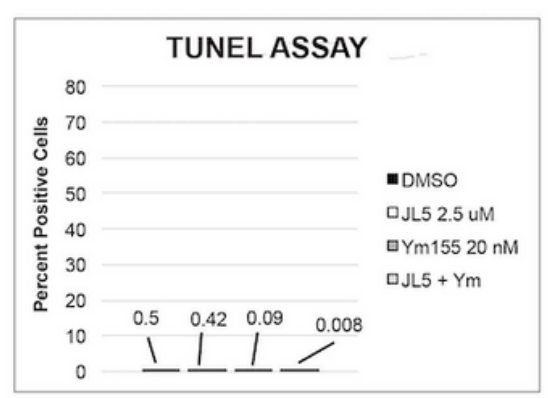

AIF Immunostaining H1299 cells

D
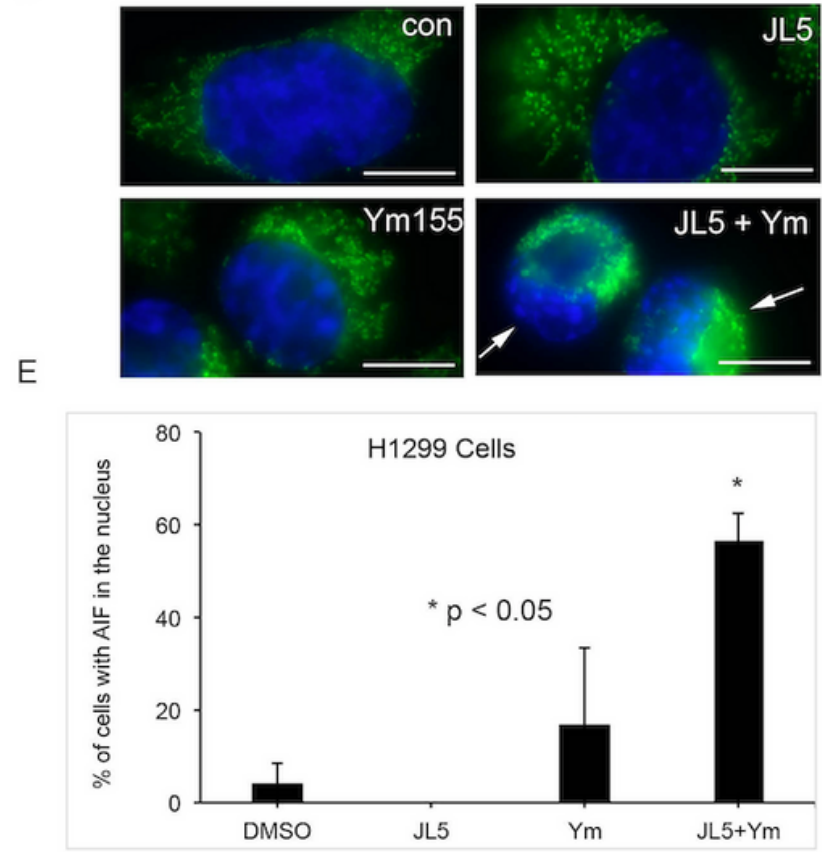

B

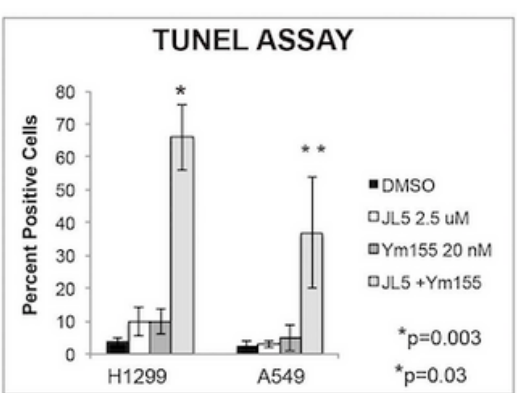

C

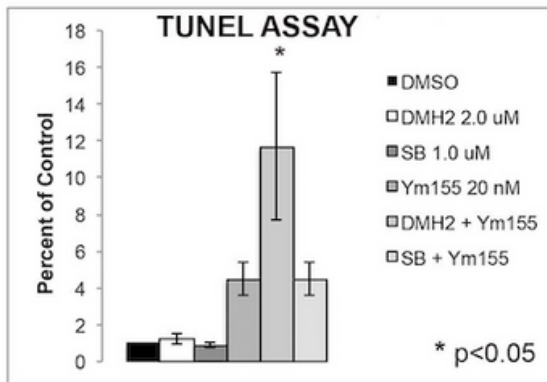

AlF Immunostaining A549 cells

$\mathrm{F}$
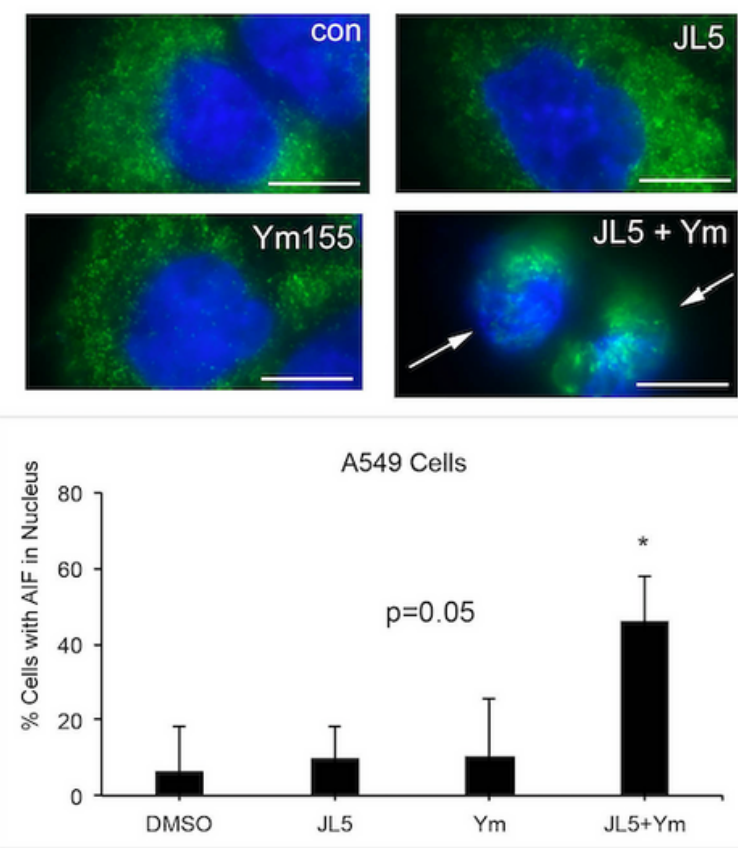

\section{Figure 4}

BMP inhibitors together with Ym155 increase DNA-DSB and AIF localization to the nucleus. (A) TUNEL assay of H1299 cells were treated with JL5 and Ym155 alone and in combination for 3 hours. (B) TUNEL assay of H1299 and A549 cells treated with JL5 and Ym155 alone and in combination for 24 hours. (C) TUNEL assay of $\mathrm{H} 1299$ cells treated with DMH2, SB-505124 (SB), alone or in combination with Ym155for 24 hours. (A-C) Data represents the mean of 4 independent experiments. (D-G) AIF Immunostaining. (D,F) Representative immunofluorescent images of AIF (green) with nucleus stained with DAPI (blue). Arrows show cells with AIF in the nucleus and condensation of the nuclei. $(E, G)$ Graphs depicting the percentage of cells with AIF localized to the nucleus for each cell line. Scale bar is equal to $10 \mu \mathrm{m}$. 
A549

A

C

BMPR2

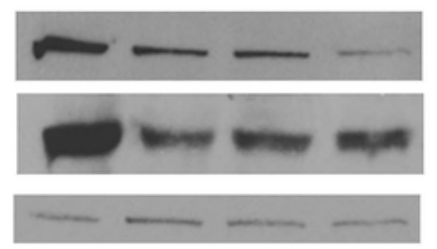

Ym155 (nM) $0 \quad 100 \quad 400 \quad 800$

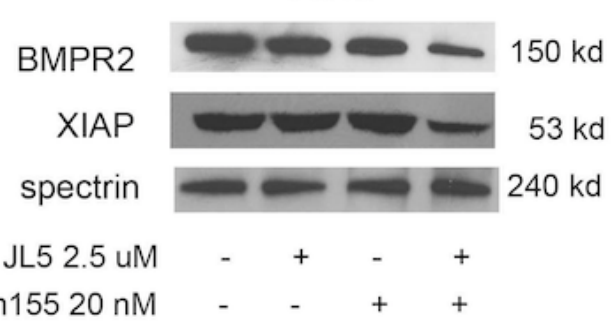

B

BMPR2

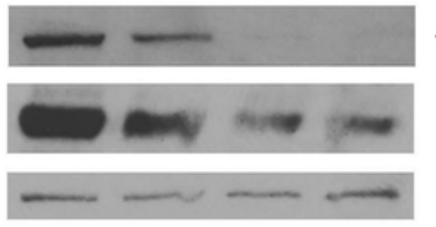

$150 \mathrm{kd}$

$53 \mathrm{kd}$

$\begin{array}{lllll}\text { Ym155(nM) } & 0 & 100 & 400 & 800\end{array}$

D

H1299

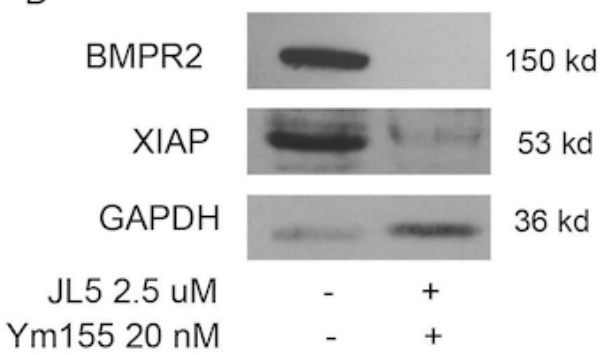

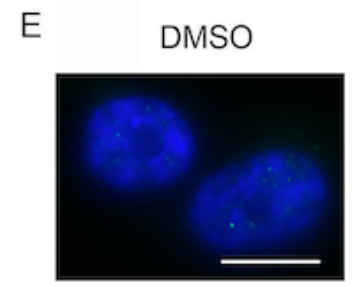
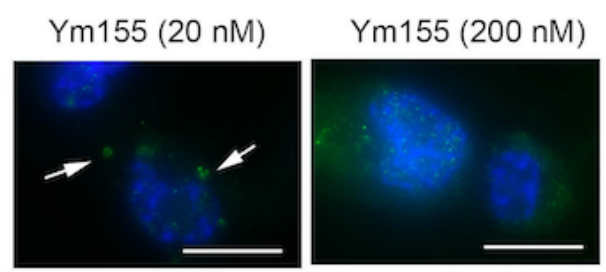

DMSO
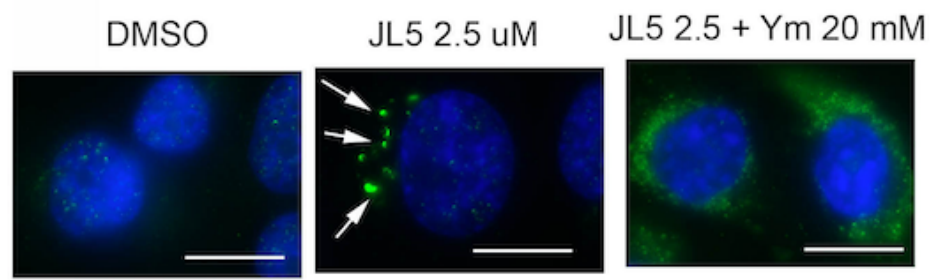
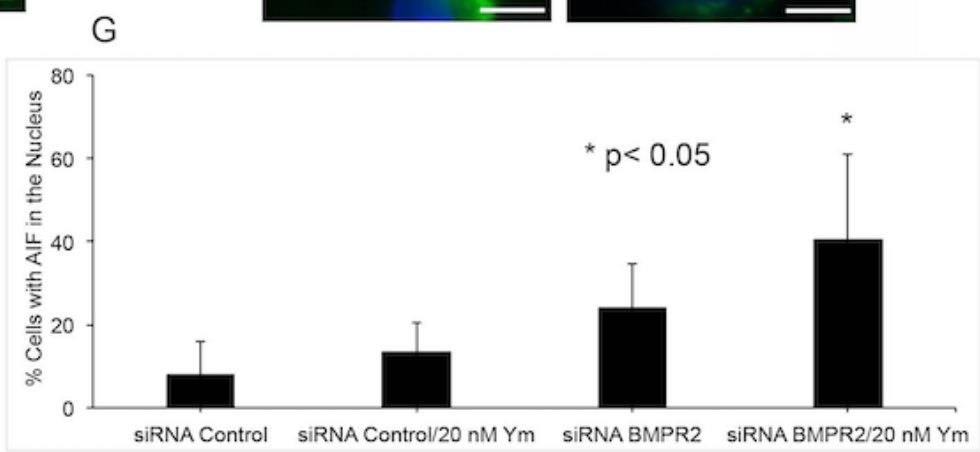

\section{Figure 5}

Ym155 regulates BMPR2 expression and causes it to localize to the cytoplasm. (A-B) Western blot of cells treated with Ym155 for 24 hours demonstrating a decrease in the expression of BMPR2 and XIAP. (C-D) Western blot of cells treated with JL5 and Ym155 for 24 hours. (E) Immunofluorescent imaging for cytoplasmic BMPR2 (green) with nucleus stained with DAPI (blue) of H1299 cells treated for 24 hours. BMPR2 localized to the cytoplasm when treated with Ym155 or JL5 $2.5 \mu \mathrm{M}$ alone, which increased significantly when compounds were used in combination. (F-G) H1299 cells were transfected with siRNA 
control or siRNA BMPR2 then treated with DMSO or Ym155 $20 \mathrm{nM}$ for 24 hours. (F) AlF localization to the nucleus increased in siRNA BMPR2 cells treated with Ym155 compared to siRNA control cells treated with Ym155. (G) Over 100 cells were quantified for nuclear localization of AIF and presented as percent positive cells. Scale bar is equal to $10 \mu \mathrm{m}$. Original Western blots are shown in Supplementary Figure 4.

A

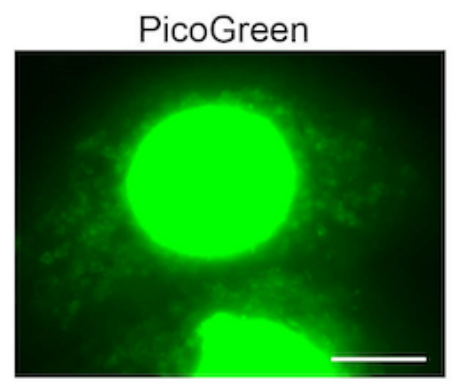

TUMF1

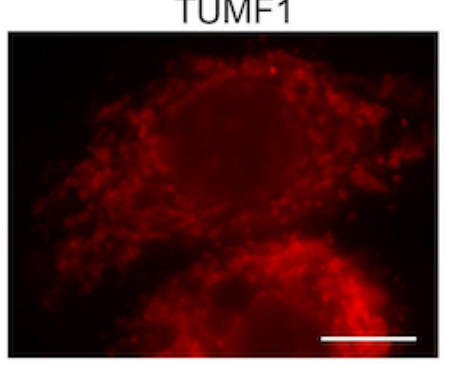

merged

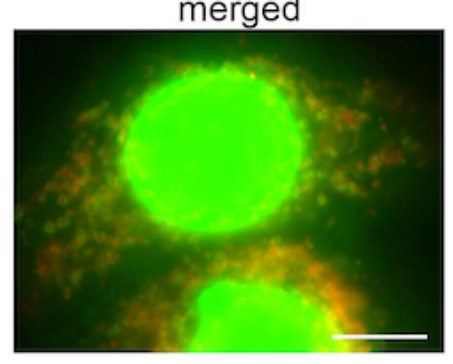

B

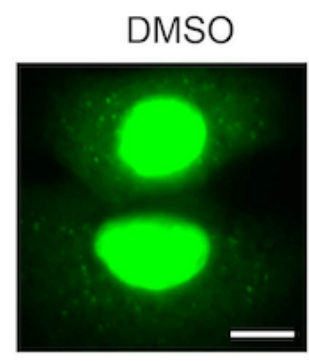

$500 \mathrm{nM}$ Ym

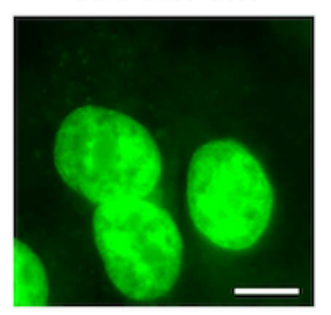

$40 \mathrm{nM}$ Ym

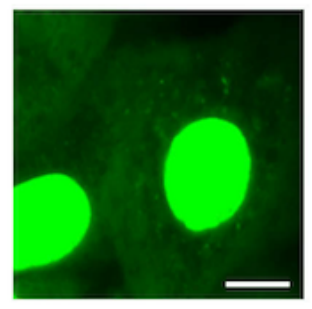

2 uM Ym

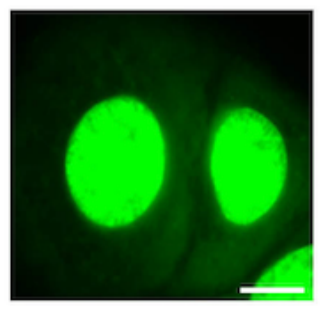

C

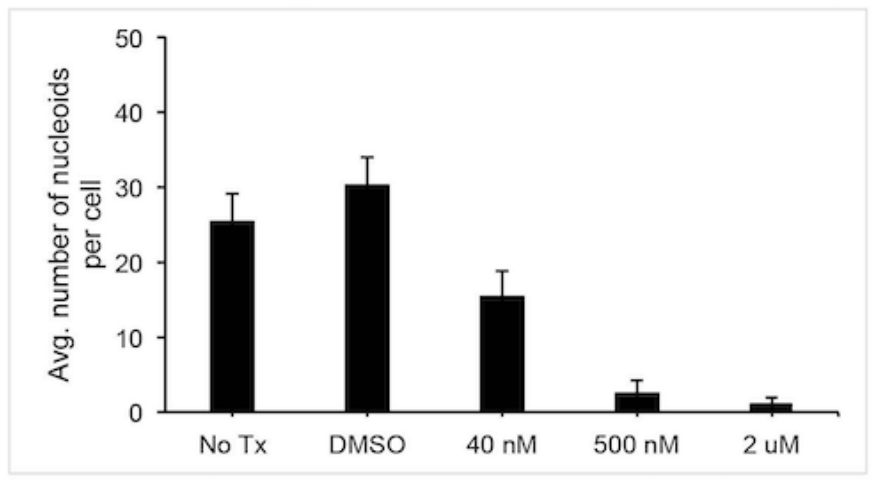

D

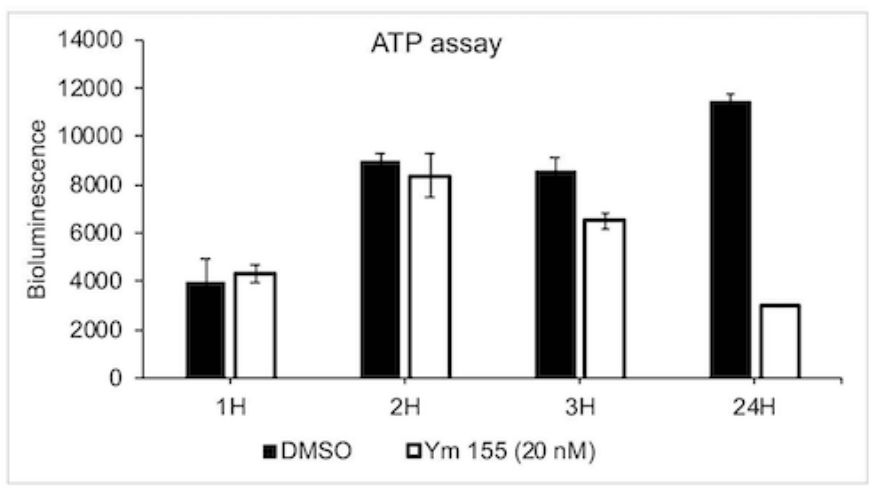

E

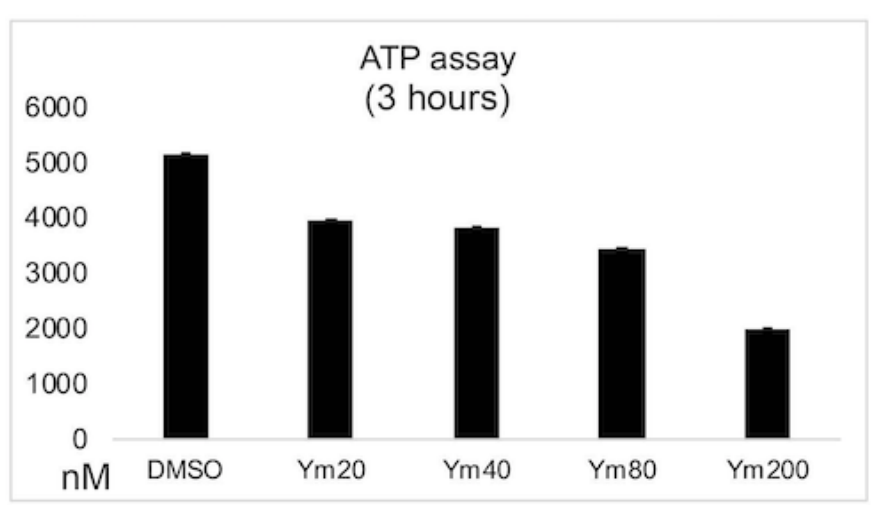

Figure 6

Ym155 binds mitochondrial DNA and decreases ATP. (A) Dual immunofluorescent imaging demonstrating co-localization of cytoplasmic PicoGreen staining and the mitochondrial marker TUMF1 in A549 cells. (B) Immunofluorescent images of A549 cells treated with increasing concentrations of Ym155 
for 1 hour then stained with PicoGreen for 1 hour. Cytoplasmic PicoGreen staining (mitochondrial nucleoids) was present in controls, which decreased with increasing concentrations of Ym155. (C) The number of mitochondria staining with PicoGreen was counted in each cell and depicted as the average number of nucleoids per cell. Approximately 100 cells were counted in each condition (D-E) Ym155 depletes ATP levels. (D) ATP levels of H1299 cells were treated with Ym155 (20 nM) for up to 24 hours. (E) ATP levels in H1299 cells treated with increasing doses of Ym155 for 3 hours. Scale bar is equal to 10 $\mu \mathrm{m}$.

\section{Supplementary Files}

This is a list of supplementary files associated with this preprint. Click to download.

- FigureS6tif.tif

- Figures2.tif

- FigureS11.tif

- Figures4.tif

- FigureS32.tif

- FigureS5New.tif 